\title{
Porosity Prediction Using Neural Network Based on Seismic Inversion and Seismic Attributes
}

\author{
Taufik Mawardi Sinaga ${ }^{1}$, M. Syamsu Rosid ${ }^{1,}{ }^{*}$, and M. Wahdanadi Haidar ${ }^{2}$ \\ ${ }^{1}$ Department of Physics, Faculty of Mathematics and Natural Science, University of Indonesia, Depok - Indonesia \\ ${ }^{2} \mathrm{PT}$. Pertamina NSB, Jakarta - Indonesia
}

\begin{abstract}
It has done a study of porosity prediction by using neural network. The study uses 2D seismic data post-stack time migration (PSTM) and 2 well data at field "T". The objective is determining distribution of porosity. Porosity in carbonate reservoir is actually heterogeneous, complex and random. To face the complexity the neural network method has been implemented. The neural network algorithm uses probabilistic neural network based on best seismic attributes. It has been selected by using multi-attribute method with has high correlation. The best attributes which have been selected are amplitude envelope, average frequency, amplitude weighted phase, integrated absolute amplitude, acoustic impedance, and dominant frequency. The attribute is used as input to probabilistic neural network method process. The result porosity prediction based on probabilistic neural network use non-linear equation obtained high correlation coefficient 0.86 and approach actual $\log$. The result has a better correlation than using multi-attribute method with correlation 0.58 . The value of distribution porosity is $0.05-0.3$ and it indicates the heterogeneous porosity distribution generally from the bottom to up are decreasing value.
\end{abstract}

Keywords: Porosity; Probabilistic Neural Network; Multi-attribute; Seismic Attributes.

\section{Introduction}

Carbonate reservoir is the main target for hydrocarbon production because carbonate reservoirs contain almost $60 \%$ of the world's total hydrocarbon reserves and are estimated to have $50 \%$ of total hydrocarbon production [1]. Hydrocarbon production can be increased if the permeability value of the reservoir is predictable, where permeability has a strong correlation with the porosity of the rock itself [2].

Porosity in the carbonate reservoir has heterogeneous and complex properties. Interpretation of seismic attributes needs to have correlation data with well data, to obtain more detailed and convincing information because of a very restricted area but better vertical resolution than seismic data. Seismic data provide better horizontal resolution and able to cover a wider area. In field, each seismic attribute has considerable uncertainty in interpretation. Therefore, by combining several attributes or implementing multiattributes can increase accuracy in predicting the reservoir property.

Neural network is an effective method used to overcome high levels of heterogeneity [3]. In this study, the neural network method is a tool used to carry out an analysis that makse a correlation between seismic data and well data. So the method can be used to predict physical property such as porosity. The neural network performs several parameters to produce output that is permissible like porosity. The best parameters that can be used as input from the neural network method are multiattributes from the integration of well data and seismic data. So, the prediction will have better accuracy.

The study location at "T" field in North Sumatera Basin. It is back-arc basin with Tertiary sediment deposited above pra-Tertiary. The focus formation is Peutu Formation (Figure 1). This formation is deposited during the Early Miocene - Middle Miocene. Peutu Formation has thick layer. It is carbonate found at the top and bottom of the formation. This formation also has sandstone at eastern region which known as Belumai Formation [4].

\footnotetext{
*Corresponding author: syamsu.rosid@ui.ac.id
} 


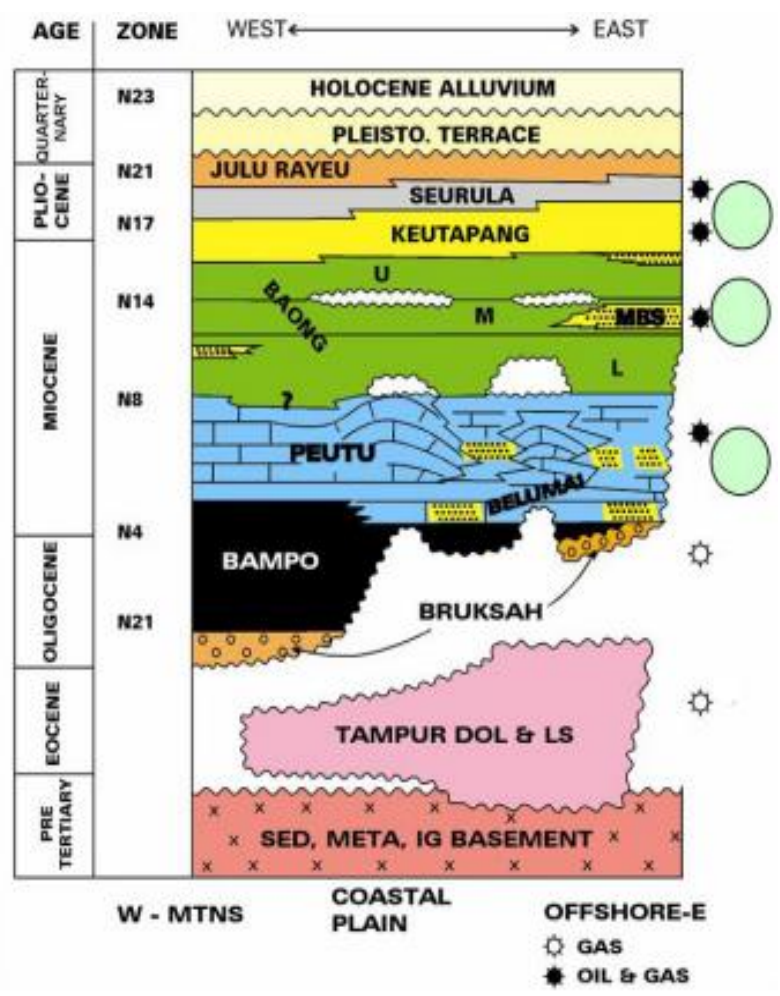

Fig. 1. Stratigraphy North Sumatera Basin [4]

\section{Theory}

\subsection{Seismic Inversion}

Seismic inversion is a method to build sub-surface model with used seismic data as input and well data as a control[5]. Seismic inversion will produce acoustic impedance parameter that will describe the better subsurface compares to conventional seismic. It is caused acoustic impedance describing subsurface layer its self while conventional seismic just describing boundary layer. Acoustic impedance is formulated as:

$$
A I=\rho * v
$$

where:

$A I=$ acoustic impedance $(\mathrm{ft} / \mathrm{s} . \mathrm{g} / \mathrm{cc})$

$\rho=\operatorname{density~}(\mathrm{g} / \mathrm{cc})$

$v=$ seismic wave velocity $(\mathrm{ft} / \mathrm{s})$

\subsection{Multi-attribute}

Multi-attribute method is one of the statistical methods that use more than one attribute to predict several physical properties of the earth [6]. In this analysis, the relationship between well data and seismic data is sought at the well location and use the relationship to predict or estimate the volume of $\log$ properties in all locations on seismic. In multi-attribute method, the best attribute selection is done to predict the target log by combining attributes with stepwise-regression. After that, obtained the best number of attributes to get correlation and for input on the neural network process [6].

\subsection{Probabilistic Neural Network}

Probabilistic neural network (PNN) is an alternative type of neural network using a mathematical interpolation [7]. PNN assumes that each new log output can be written as a linear combination of $\log$ values in the training data. In this study, PNN is a method can make nonlinear equations to get values with better correlation and a higher resolution. PNN can be written using equations:

$$
\begin{gathered}
\{\mathrm{A} 11, \mathrm{~A} 21, \mathrm{~A} 31, \mathrm{~L} 1\} \\
\{\mathrm{A} 12, \mathrm{~A} 22, \mathrm{~A} 32, \mathrm{~L} 2\} \\
\{\mathrm{A} 13, \mathrm{~A} 23, \mathrm{~A} 33, \mathrm{~L} 3\} \\
\ldots \\
\text { AA1n, A2n, A3n, Ln }
\end{gathered}
$$

$\mathrm{n}$ is training data and there are three attributes to be used. $L_{i}$ is target log will be predicted as every training example. PNN assume that each new value of the new log output can be written as a linear combination of log values in the research data. To sample new data with attribute values can be written:

$$
x=\left\{A_{1 j}, A_{2 j}, A_{3 j} \ldots A_{n j}\right\}
$$

Then, a new $\log$ value is calculated with the following equation

$$
L^{\prime}(x)=\frac{\sum_{i=1}^{n} L_{i} \exp \left(-D\left(x, x_{i}\right)\right.}{\sum_{i=1}^{n} \exp \left(-D\left(x, x_{i}\right)\right.}
$$

where

$$
D\left(x, x_{i}\right)=\sum_{j=1}^{n}\left(\frac{x_{j}-x_{i j}}{\sigma_{j}}\right)^{2}
$$

$D\left(x, x_{\mathrm{i}}\right)$ is the distance between the input data and each training point $x_{i}$. Parameter $\sigma_{j}$ is a normalize from distance that measured in a multidimensional attribute space $^{[6]}$.

Equations (4) and (5) describe the application of the PNN algorithm. Then, the result of validation using PNN is given in equation (6):

$$
L_{m}^{\prime}\left(x_{m}\right)=\frac{\sum_{i \neq 1}^{n} L_{i} \exp \left(-D\left(x_{m}, x_{i}\right)\right)}{\sum_{i \neq m}^{n} \exp \left(-D\left(x, x_{i}\right)\right)}
$$

The equation is used to get the validation results for the sample target to $m$. Prediction of the validation value of the sample target to $m$ is done when the sample is not calculated in the training data. By repeating this process for each training sample. then, total error prediction is obtained by the equation :

$$
E_{y}\left(\sigma_{1}, \sigma_{2}, \sigma_{3}\right)=\sum_{i=1}^{N}\left(L_{i}-L_{i}^{\prime}\right)^{2}
$$

The results obtained through PNN analysis will form a nonlinear transformation between the target $\log$ and seismic attributes in the cross plot diagram. The results of non-linear transformations expect a better correlation value between the actual log and the log model compared to the results of linear transformations generated through a decrease in multi-attributes. 


\section{Methodology}

This study uses 2 wells and 2D post-stack time migration (PSTM) seismic data which aims to predict total porosity using PNN method.

This study is finding one best attribute by trial and error. After that, using stepwise regression to find some best attribute based on lowest validation error. Next, prediction porosity based on attributes have been selected to be used as input PNN processing by non-linear, the architecture of PNN has been explained in point 2.3.

\section{Results and Discussion}

\subsection{Well Data Analysis}

Well data (Figure 2) from 9570 - $9870 \mathrm{ft}$ show that the value of density and P-wave have been increasing but the gamma-ray has been decreasing. Value of P-wave increase, show that $\mathrm{P}$-wave through compact medium. It also occurs in density. When value of density increase, it is indicating the compact zone. Lithology which has the character of compact rock is carbonate.

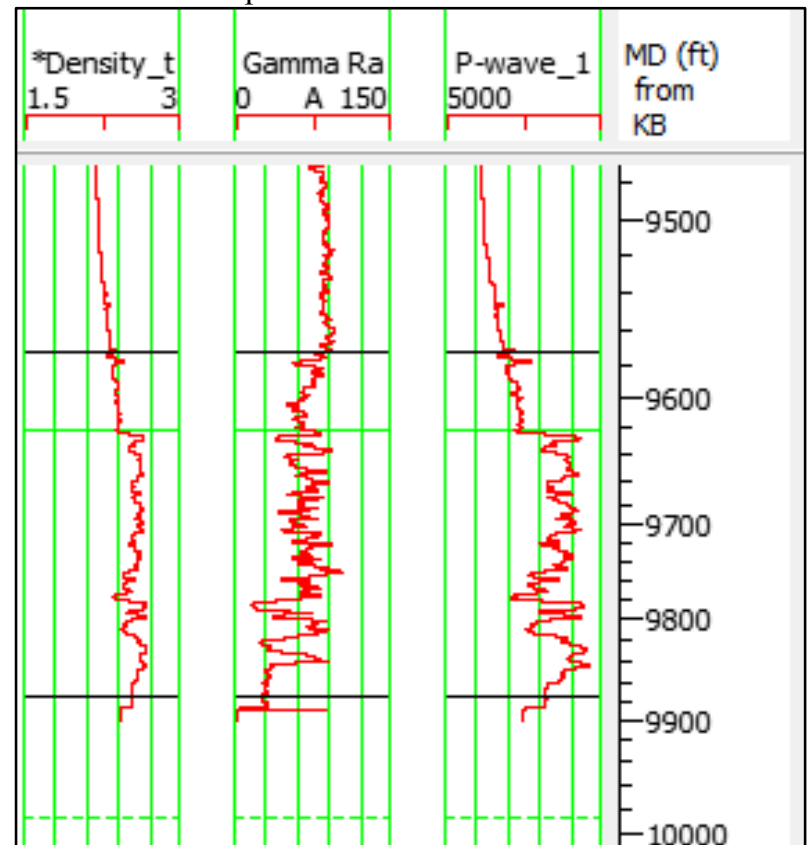

Fig. 2. Well data of C1 which consist of GR, Density and PWave to Depth

\subsection{Acoustic Impedance}

Acoustic impedance is gotten by inversion seismic. Inversion seismic is used model-based method because model-based has better correlation than other seismic inversion methods. Inversion result shows impedance value in the target zone of this study (Figure 3). Alteration of acoustic impedance value can be interpreted as porosity value. When acoustic impedance is high, porosity value is low. Acoustic impedance will be used as an attribute for the multi-attribute process. Acoustic impedance as attribute to multi-attribute process selection.

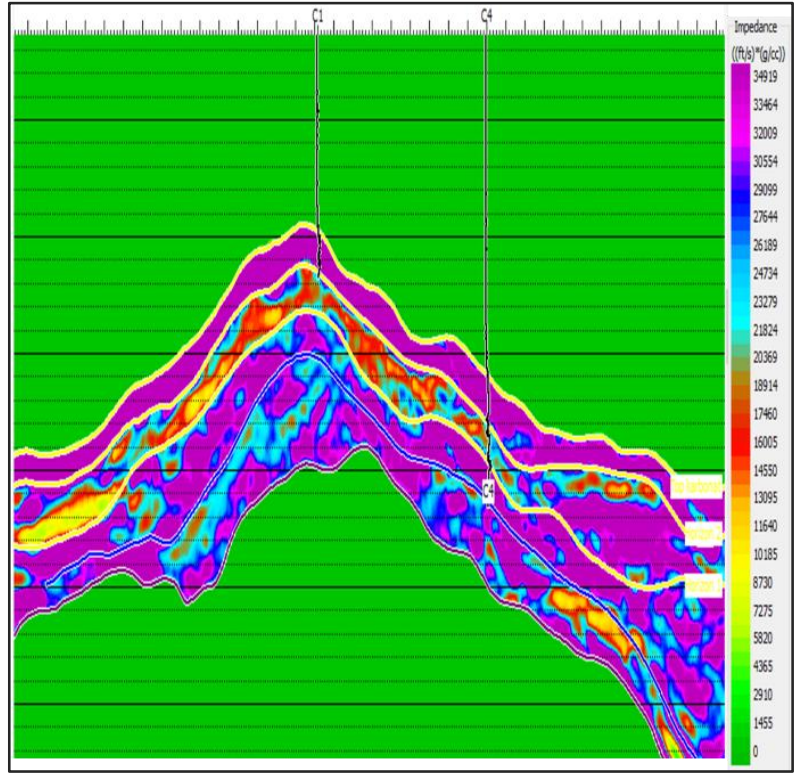

Fig. 3. The Results Of The Post-Stack Inversion Model-Based

\subsection{Multi-attribute}

Table 1 shows the list of seismic attributes, with corresponding prediction errors and correlation coefficients. Amplitude envelope is the best attribute with correlation 0.52 to predict porosity. It has high value is indicating hydrocarbon contains.

Table. 1. List Of Attributes On Single Attribute

\begin{tabular}{|c|c|c|c|c|}
\hline & Target & Attribute & Error & Correlation \\
\hline 1 & Porosity & Amplitude Envelope & 0.070090 & 0.525800 \\
\hline 2 & $(\text { Porosity })^{\star \star 2}$ & Amplitude Envelope & 0.070895 & 0.527905 \\
\hline 3 & Sqrt( Porosity) & Amplitude Envelope & 0.071521 & 0.485299 \\
\hline 4 & Porosity & Amplitude Weighted Frequency & 0.074078 & 0.437928 \\
\hline 5 & $(\text { Porosity })^{\star \star 2}$ & Amplitude Weighted Frequency & 0.075492 & 0.409376 \\
\hline 6 & Sqrt( Porosity) & Amplitude Weighted Frequency & 0.075530 & 0.426110 \\
\hline 7 & Porosity & Amplitude Weighted Phase & 0.080721 & -0.200819 \\
\hline 8 & Porosity & Integrated Absolute Amplitude & 0.081234 & 0.167627 \\
\hline 9 & Porosity & (Inversi_Terbaru_Zp $)^{\star \star 2}$ & 0.081532 & 0.144727 \\
\hline 10 & Sqrt( Porosity) & Amplitude Weighted Phase & 0.081645 & -0.180477 \\
\hline 11 & Porosity & Inversi_Terbaru_Zp & 0.082077 & 0.088416 \\
\hline 12 & Sart( Porositv) & Intearated Absolute Amolitude & 0.082190 & 0.171867 \\
\hline
\end{tabular}

Figure 4 shows Log model use amplitude envelope has a high difference from the actual porosity and the correlation value is low. Therefore, to get a higher correlation and log model closer to the actual log, it is necessary to combine attributes. 


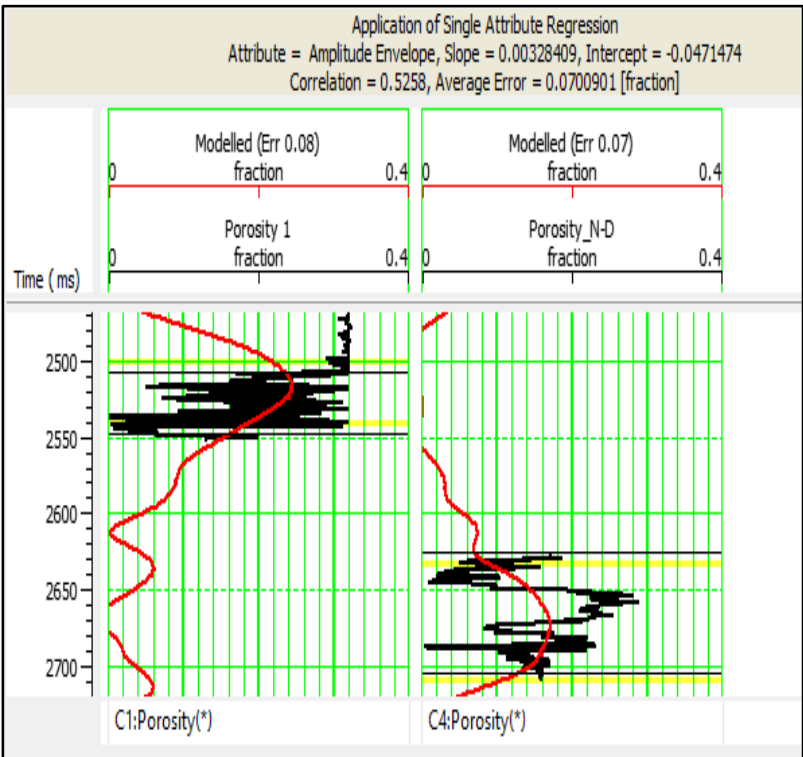

Fig. 4. Log Model with Best Single Attribute vs Log Actual

List of attributes have been selected by using multiattribute as shown in Table 2. Multi-attribute process is carried out using step-wise-regression. The first step is finding the best single attribute and it has been found in previous stage (Table 1). After that, combine the best single attribute with other attributes to find second attributes have minimum errors and so on.

Table 2. List Of Attribute Used Multi-attribute

\begin{tabular}{|l|l|l|l|l|}
\hline \multicolumn{1}{|c|}{ Target } & \multicolumn{1}{|c|}{ Final Attribute } & \multicolumn{1}{c|}{ Training Error } & \multicolumn{1}{c|}{ Validation } \\
1 & Porosity & Amplitude Envelope & 0.069832 & 0.177340 \\
\hline 2 & Porosity & Average Frequency & 0.061558 & 0.122855 \\
\hline 3 & Porosity & Amplitude Weighted Phase & 0.059567 & 0.184058 \\
4 & Porosity & Integrated Absolute Amplitude & 0.057620 & 0.167178 \\
\hline 5 & Porosity & (Inversi_Terbaru_Zp ) & 0.052681 & 0.273129 \\
\hline 6 & Porosity & Dominant Frequency & 0.051164 & 0.117304 \\
\hline 7 & Porosity & Amplitude Weighted Cosine Phase & 0.050440 & 0.165645 \\
\hline 8 & Porosity & Instantaneous Frequency & 0.050149 & 0.155152 \\
\hline 9 & Porosity & Amplitude Weighted Frequency & 0.048584 & 0.334307 \\
\hline 10 & Porosity & Raw Seismic & 0.048417 & 0.336403 \\
\hline
\end{tabular}

Based on Figure 5, the black curve is the error calculated using the training data and the red curve is the error calculated using the validation data. The red curve describes sixth attributes has low error 0.11 and occurred increase error validation value on seventh attribute until tenth attribute. The increase of validation error value conduces to decrease correlation value. Attributes are used in multi-attribute process include amplitude envelope, average frequency, amplitude weighted phase, integrated absolute amplitude, acoustic impedance, and dominant frequency.

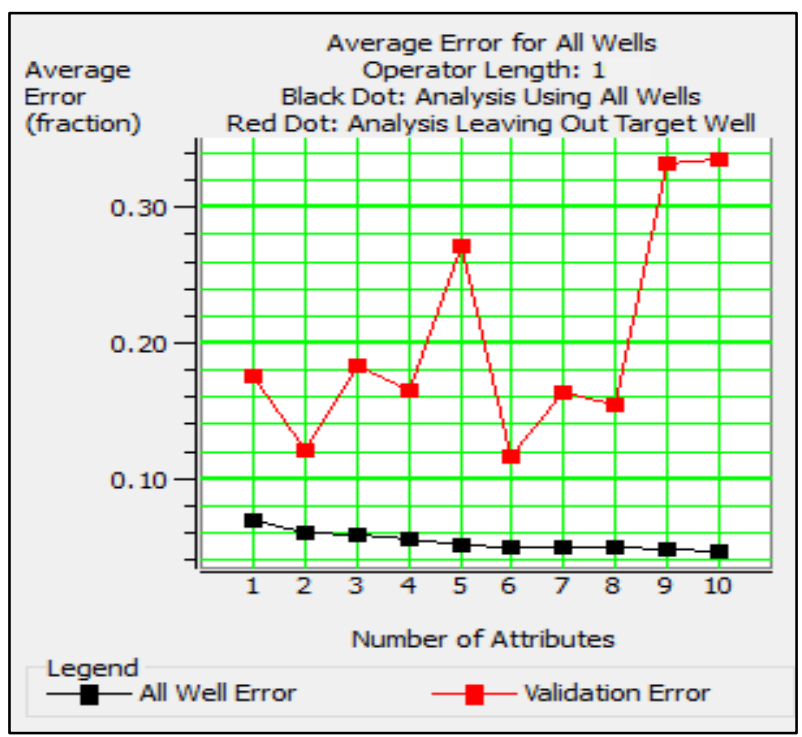

Fig. 5. Validation vs. Prediction Error Curve

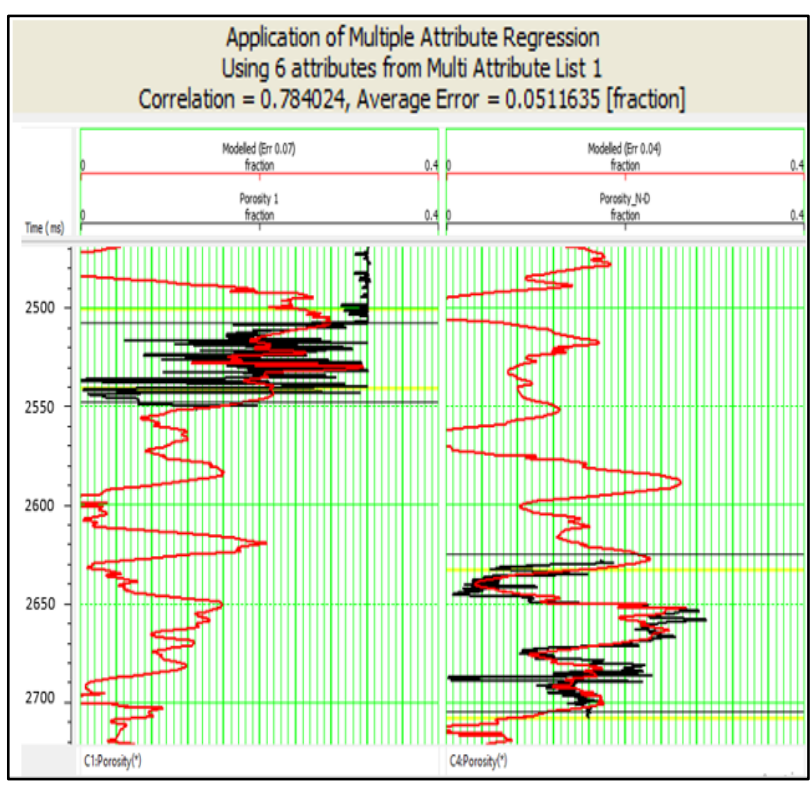

Fig. 6. Log Model vs. Log Actual Using 6 Attributes

The best six attributes have been selected will be applied to log curve (Figure 6). The curve log shows prediction log (red line) and actual log (black line) is different. The difference is caused by multi-attribute using linear equation to predict physic property. While, seismic attribute and data well are non-linear, Therefore, nonlinier method is needed to get best correlation and prediction log approaches the actual log.

\subsection{Probabilistic Neural Network to Predict Porosity}

In the previous process, prediction of porosity values was carried out using multi-attribute analysis which obtained a relatively low correlation coefficient. For this reason, non-linear methods are used to increase the correlation coefficient and increase the prediction log similarity with the actual $\log$. 


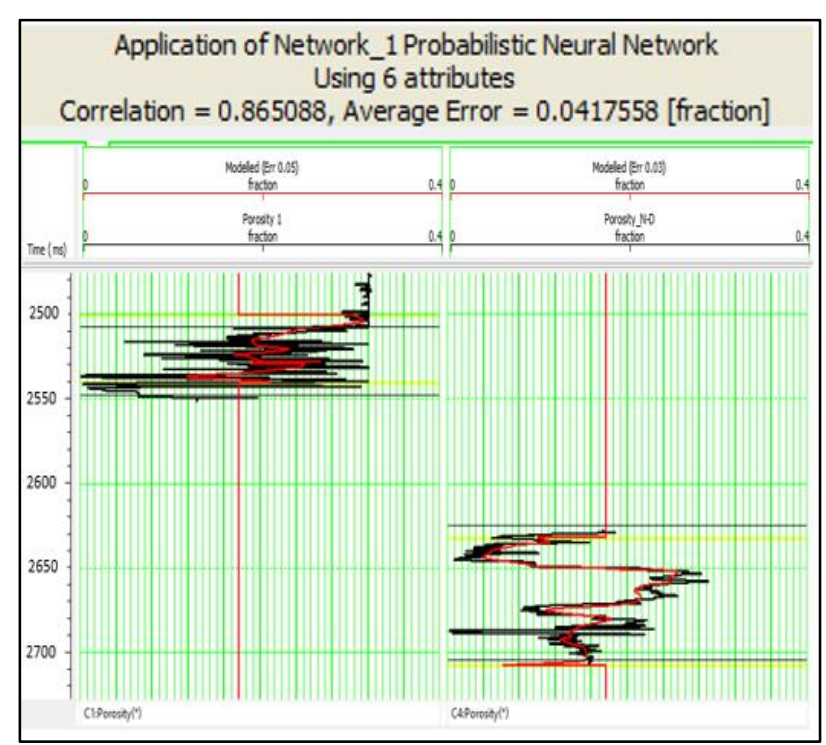

Fig. 7. Log Model PNN vs. Log Actual

The prediction results of total porosity using PNN are similar to the actual log and the value of the correlation coefficient is 0.86 (Figure 7). The high correlation coefficient value proves that PNN is better than the multiattribute method. Because, PNN is a method that can produce nonlinear equations to get predictive results obtained like actual log.

Total predictive porosity $(\log )$ of the PNN results distributed to seismic data to build porosity volumes (Figure 8). The value of distribution porosity is in range $0.05-0.3$.The high porosity in reef structure $\mathrm{C} 1$ is located at Top Formation $(0.2-0.3)$. While, reef structure $\mathrm{C} 4$ at Top Formation is lower porosity than $\mathrm{C} 1(0.05-0.13)$ and the porosity value is caused by overburden. So, carbonate at reef structure $\mathrm{C} 4$ is dominated by Mudstone to Wackestone. Because, C4 located at slope with low energy condition. In other hand, reef structure $\mathrm{C} 1$ is located at core reef which show carbonate dominated is grainstone - rudestone. It cause high value of porosity.

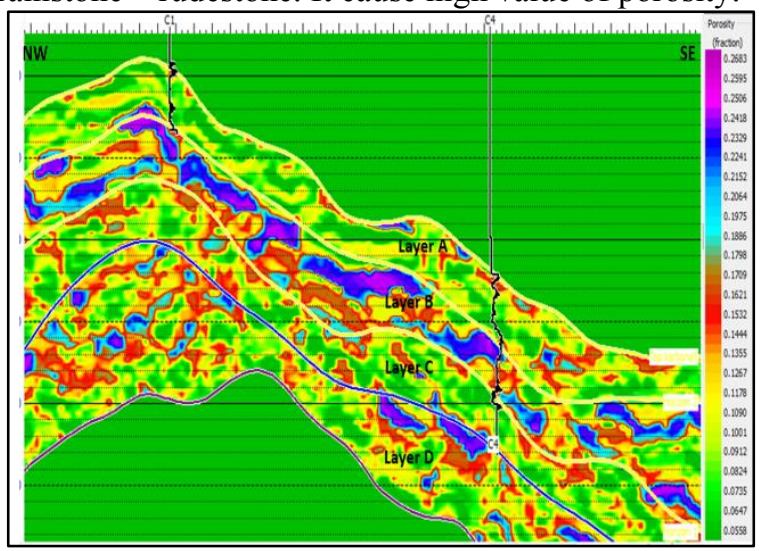

Fig. 8. Porosity Cross Section from PNN

\section{Conclusion}

Probabilistic neural network method used 6 best attributes obtained high correlation 0.86 and getting log prediction approach log actual. This result has better than using multi-attribute method with correlation 0.78. Base on porosity distribution in seismic can be analyzed have porosity value $0.05-0.26$. Porosity distribution in layer $\mathrm{B}$ has higher porosity value than other layers. High porosity in layer $\mathrm{B}$ is indicating that has hydrocarbon potency.

\section{References}

1. S. Xu, M. A. Payne, Modeling Elastic Properties in Carbonate Rocks. The Leading Edge, 28, 66-74. (2009)

2. L. Zhao, M. Nasser, D. Han, Quantitative Geophysical Pore-Type Characterization and Its Geological Implication in Carbonate Reservoirs. European Association of Geoscientists \& Engineers, Geophysical Prospecting, 1- 15. (2013)

3. T. Mori, E. P. Leite, Porosity Prediction of a Carbonate Reservoir in Campos Basin Based on the Integration of Seismic Attributes and Well Log Data. (2018)

4. R. Cameron, M.C.G. Clarke, D.T. Aldiss, J.A.Aspden, A. Djunuddin, The Geological Evolution of Northern Sumatera. (1980)

5. S. Sukmono, Seismik Inversion and AVO Analysis For Reservoir Characterization, Departemen Teknik Geofisika ITB, Bandung (2002)

6. D. Hampson, J. Schuelke, J.A Qurein, Use of Multiattribute Transforms to Predict Log Properties from Seismic Data, Geophysics, 66,p.220-236 (2001)

7. D.F.Specht, Probabilistic neural network, Neural Networks, 3( 1) :109-118.(1990) 